\title{
RELATIONSHIP OF CONDITION INDEX AND REPRODUCTIVE STATUS OF BLACK SNAIL, Fannus ater IN MERCHANG LAGOON
}

\author{
NURUL HUSNA HASSAN ${ }^{A^{*}}$ AND ROSLIZAWATI AB LAH \\ ${ }^{\mathrm{a}, \mathrm{b}}$ Faculty of Fisheries and Food Science, Universiti Malaysia Terengganu, Kuala Nerus, Terengganu, Malaysia \\ *Corresponding author: husnahassan9561@gmail.com
}

\begin{abstract}
Black snail, Faunus ater is an abundant species in Malaysia yet not many research have focused on its physiological and biological activities. This research aimed to assess the condition index (CI) and reproductive status based on the gonadosomatic index (GSI) for short-term duration. Samples were collected monthly from Merchang Lagoon, from November 2018 to January 2019. Four different types of condition indices equation were applied in this study and the results revealed that there were significant differences between four equations for measuring the $\mathrm{CI}(\mathrm{P}=0.000)$. However, the result for the GSI shows no significant difference between three month of sampling $(\mathrm{P}>0.05)$. CI based on fresh weight measurement (Fww/Tww x 100) and dry weight measurement (Fdw/Fww x 100) reached its peak when GSI decreased. The rest of the trend for CI fluctuated and CI was not affected by GSI. Overall, this study concluded that, there were trends observed in CI and GSI for the black snails. However, it is suggested that longer term observation in future research is needed have a better understanding on the black snails.
\end{abstract}

Keywords: Black snail, Condition index, Faunus ater, Gonadosomatic index

\section{Introduction}

Black snail, Faunus ater or known as devil snail has features of smooth, slender, high-spired black shell, unusual aperture with two deep sinuses, and the feature consists of anterior and anal canals ( Houbrick, 1991; Lok et al.,2011). It is an abundance species in Malaysia and widely distributed from fresh to slightly brackish water in the Indo-West Pacific including Singapore and they were found to clog some drains ( Brandt, 1974; Houbrick, 1991; Lok et al., 2011). Literatures on ecological studies of $F$. ater have been found. The vast number of $F$. ater in the ecosystem shows that this species is underutilized and not commercially exploited for any purpose in Malaysia (Das et al., 2018). To date, this species is consumed as human food sources in several countries by the local people as it is can be found easily in those regions ( Houbrick, 1991; Lok et al., 2011). However, due to fewer studies on this species the numbers of references available is limited.

Unfortunately, there is a depth of studies on the physiological activities of the species can be making it difficult to find references on the physiological activities such as growth, reproduction, and excretion, among others of this species ( Lucas \& Beninger, 1985; Galvao et al., 2015). Therefore, more studies are needed in order to understand the reproductive status of F. ater.

Several ways have been put in place in order to measure the physiological and biological conditions of the species such as CI and GSI. Several equations have been applied for calculating the CI which can be calculated based on the dry weight or fresh weight. The alternative way to observe the reproductive cycle of certain species is by measuring the condition index (CI) of the organisms. Condition index is a method which facilitates in determining the health status of animals including gastropod. This method is an indirect way to observe the reproduction of $F$. ater. Moreover, there are several available studies on CI on other species of gastropod and several attempts have been made using different methods of calculating the CI including: dry shell weight or wet weight, dry flesh weight or wet weight to calculate the CI. However, fewer studies on comparing equations are available and have not practically indicated 
the suitable CI equations to be used in order to examine the health status and for implementing a suitable $\mathrm{CI}$ to be used for measuring the status of the gastropods. CI may influence the reproductive stages of the gastropod. In order to check the validity of the CI method, GSI measurements need to be done. Measurements of gonad size and digestive gland vary between species. Therefore, it is important to compare all the available equations for measuring the CI together with the GSI in order to observe if there is any positive or negative trend within this parameter.

Additionally, it is important to have clear information on the reproductive cycle of organisms in order to establish adequate fishery management measures which contribute to sustainable exploitation of resources. On the other hand, other species which are not commercially exploited like Faunus ater have high possibilities of invading the ecosystem. Thus, it is important to prevent them from occupying the area and form any undesirable ecosystem destruction. Even though $F$. ater is abundance, there is no evidence showing that this species could be a harmful organism to the ecosystem. The main concern is to acknowledge the abundant of $F$. ater in the ecosystem and how to determine the reproductive status of this species. The massive number of $F$. ater might indicate the status of the ecosystem itself.

Thus, this study is essential in order to determine the reproductive status of $F$. ater by measuring their condition index based on several methods that are available in the literature. To assess the condition index of Faunus ater based on different equations and observe the reproduction status of Faunus ater for shortterm duration. Meanwhile, comparison of the condition index with reproductive status will be done to see the relationship between these two methods referring to various journals and articles that are available. The data will provide available information on current reproductive status of $F$. ater based on GSI. By measuring GSI, it will clearly distinguish the pattern of reproductive activities of the gastropod. Consequently, this study will be a baseline for further studies which relate to the condition of $F$. ater.

\section{Materials and Methods}

\section{Sampling}

This study was conducted in Merchang Lagoon in the state of Terengganu which is situated the in eastern part of Malaysia. Samples (Figure

1) were collected from Merchang Lagoon for three consecutive months from November 2018 to January 2019. Throughout the sampling period, observations of the sites were taken into account to study the surrounding areas, association of organisms in the sampling area and to identify the community activities which could be the factors contributing to the changes of physiological and biological aspects of the species. All samples were place in a pail and brought back to the laboratory to be processed. Sealed plastic bags were used to keep samples in the freezer for further analysis.

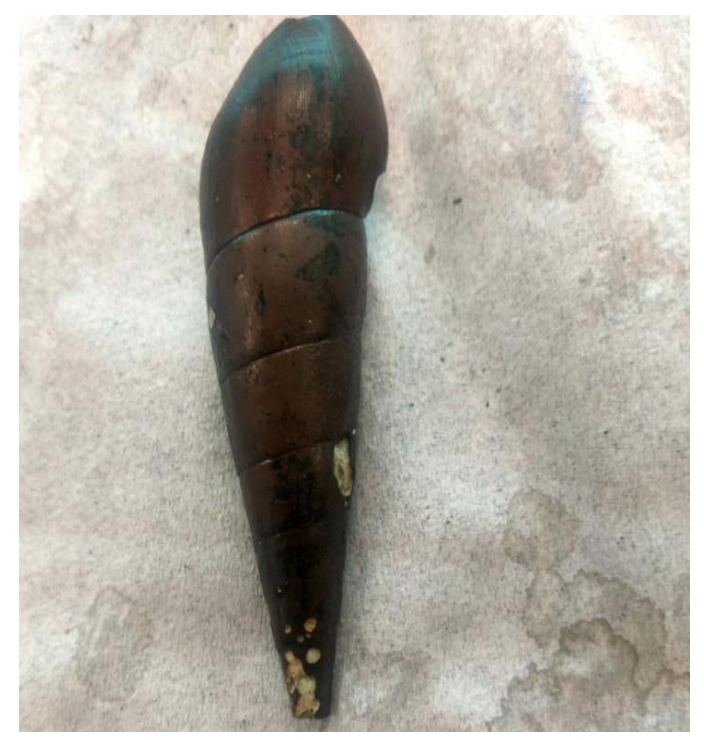

Figure 1: Sample of black snail, Faunus ater (Photograped by Nurul Husna)

\section{Laboratory works}

Each sample was washed under running water with a brush to eliminate the small barnacles, 
mold and excess slime from the shell of the samples. Samples were dried to reduce and remove excess water from shell and flesh using absorbent paper. Drying the sample using absorbent paper gave out weight different results and were varied in conjunction with the water content each sample held. The measurement of the shell length (SL) was taken by using vernier calliper and the digital balance has been used to measure the total weight (Tw). Total weight of each sample was recorded for assessment. Deshelling process using bench vice was carried out after the measurement of shell length and total weight was taken. The purpose of de-shelling was to remove the flesh from the shell and to eliminate excessive water from internal part of the gastropod to get the total flesh wet weight (Fww). The flesh and shell were separated for future assessment on CI and the measurement of gonad was taken for GSI analysis.

\section{Conventional condition indices}

Different authors have measured condition index (CI) and gonadosomatic index (GSI) in a variety of ways. In order to calculate conventional condition indices and gonadosomatic index, a total of 50 individuals with length of $41-68 \mathrm{~mm}$ SL were sampled for three months. The exact similar species were used to estimate the GSI. The flesh and shell wet weights were measured using digital balance. In order to get the flesh wet weight, all broken shell from the process of de-shelling were collected to make sure that the wet weight was not affected by the broken fragments. Then the flesh wet weight was measured. To get the dry weights of the flesh and shell, these two major components were dried at $100^{\circ} \mathrm{C}$ for 2 days for the duration of 48 hours. After 48 hours, the dried samples were taken out from the oven and allowed to cool for 10-15 minutes before the flesh dry weight (Fdw) and shell dry weight (Sdw) was measured using digital balance. The procedures were repeated each month for different batched of samples and it took a total of approximately 6 days 144 hours to get the constant weight of dry flesh and shell of all samples. The calculation was done following that of Vasconseles et al., 2008 and in this study on CI were: Fww/Tw x 100, Fww/ Sww x 100, Fdw/Fww x 100 and Fdw/Sdw x 100.

\section{Gonadosomatic index}

Samples of gonad and digestive glands were taken from the same specimens of 50 individuals collected in November 2018 to January 2019. Gonadosomatic index (GSI) was calculated using weight of gonad and digestive gland. The total weight of the flesh was taken into account: Ggd/Tww x 100 where Ggd is gonad gland and digestive gland while Tww is total wet weight. The method of processing the gonad and digestive gland was centralized in order to eliminate bias and uneven data. The gonad was located right below the flesh in the middle. The Ggd was cut right before the gonad and then separated from the flesh. Standardization of area to be cut was made in order to eliminate any possible bias which can affect the weight and results. All raw data on length, wet and dry weights of the $F$. ater, was recorded in table using Microsoft Excel. The data was then used in order to run the statistical analysis.

\section{Statistical analysis}

Statistical analysis was conducted using minitab18 software. Values of CI and GSI were recorded as mean and standard deviation. All data were first tabulated in a table and by using one-way analysis of variance (ANOVA) the significant level between each CI as well as between GSI and months were tested for each set of data. Level of significant difference was set at $5 \%$ for the test. When ANOVA detected significant results in CI, the Dunns' Post Hoc test was then performed. Dunns' Post Hoc test was used to determine the significant difference among condition indices. The significance of all CI was tested and the $\mathrm{p}$ value for Post Hoc Test determined according to the number of CI methods. In addition, the significant differences (Dunns' test) between the first and the second highest CI values in relation to the CI values of the following months were verified. 


\section{Results and Discussion}

\section{Conventional condition index}

The correlation between each condition index and gonadosomatic index were tested and the results were shown in Figure 2. In this study, Figure 2 presents the results obtained from preliminary analysis of the trends between condition index (CI) and gonadosomatic index (GSI). In the same figure, the chart above it represents the results for $\mathrm{CI}$ while the lower chart has the results for GSI. Clear patterns of each CI were recorded according to the month in which it can be distinguished as seen in Figure 2. About the condition index, similar pattern was shown by CI1 and CI2 (Dunn's Test, P<0.05). For CI1 and $\mathrm{CI} 2$, the initial mean value started with the lowest at 50.33 and 10.54 in November 2018. Meanwhile, in December 2018, the mean values of both CI were at the peak of where the value of CI1 was 64.68 and CI2 was 16.87 respectively. The patterns of condition indices were slightly fluctuated during the month of January 2019 with the value of 64.61 for CI1 and 16.58 for $\mathrm{CI} 2$. Thus, the similarities of pattern and trend were shown between these two CIs and made it clear to distinguish from other CId. What is interesting with the data is that, the mean values of CI1 were much greater compared to the mean values of CI2, even though both were showing a similar pattern.

On the calculation for dry weight and wet weight of the flesh and shell in CI3 and CI4 $(\mathrm{P}<0.05)$, the pattern was completely different, showing the negative correlation between CI1 and CI2. In Figure 2, CI3 showed the highest mean value of CI in November 2018 with the value of 31.04 and the lowest value was recorded on December 2018 with the value of 26.79. In January 2019, the mean value increased slightly. To date, a completely different pattern was shown in CI4 as the pattern increased starting from November 2018 to January 2019 without a slight decrease of value throughout the sampling period. This result might be intact, in conjunction with the calculation method which compromised dry flesh and shell weight. The lowest value was recorded in November 2018 with a mean value of 3.21 , while the value began to increase to 4.62 in December 2018 and in January 2019 it was at the peak, 4.84. The overall results of CI3 and CI4 were showing an opposite pattern to the $\mathrm{CI} 1$ and $\mathrm{CI} 2$ during these particular periods of the year.

\section{Gonadosomatic index}

The gonads were examined externally in order to see the size distinction of the gonad in each month during the study period. The biggest gonad sizes were from the samples of November 2018. In the same figure, the results indicated the trend of GSI for these three months of sampling period with no significant difference $(\mathrm{P}>0.05)$ when using month as the main factor for comparison. It was difficult to differentiate the sexes as there are no available references on the reproductive system of $F$. ater. In November 2018, the plotted chart for GSI was presented a highest value of the index when compared to the value during December 2018 and January 2019. The value during the period of December 2018 seems to have dropped significantly and resulted in the lowest GSI, while in January 2019 the GSI began to increase. A clear comparison between these results was presented in the graph using mean value and standard deviation data. The patterns and trends of both CI and GSI can be identified in Figure 2 for references.

Overall, the results have clearly shown the differences between CI and GSI trends within three months of experimental execution. In reviewing the literature, no data was found on the association and relationship between condition index and gonadosomatic index of $F$. ater. In this study, the CI was a study to allocate the similarities and dissimilarities of trends when compared to GSI in order to find the best correlation of CI and GSI in which the output will aid in the current knowledge on how certain methods of CI can be used to determine the fecundity of $F$. ater as an alternative way to GSI. 

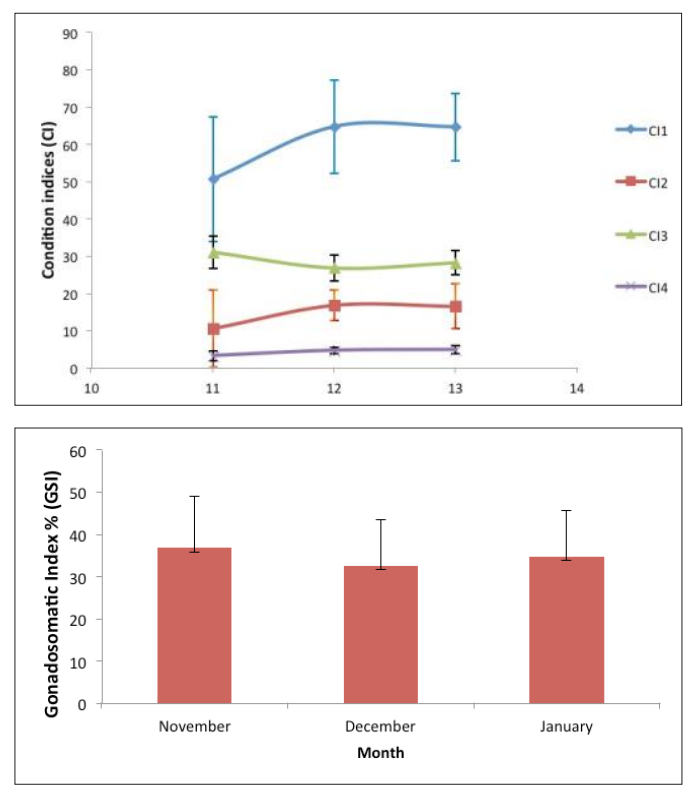

Figure 2: Upper chart shows the variation of condition indices (CI) of Faunus ater on monthly collection and lower chart shows the trend of GSI during the sampling period. Error bars in both charts symbolize the standard deviation and symbols at the side of the CI graph represent the mean of significant difference of CI. CI1 indicate flesh wet weight / total wet weight (Fww / Tww), CI2 flesh wet weight/ shell wet weight (Fww/Sww), CI3 was flesh dry weight/flesh wet weight (Fdw/Fww) and CI4 is flesh dry weight/ shell dry weight (Fdw/Sdw)

Significance of these CI was approved by using analysis of variance one way (ANOVA one-way), given value $(\mathrm{P}<0.05)$. There was significant difference between the CIs. In CI1 and CI2 (Dunn's Test, $\mathrm{P}<0.05$ ), the trend was one opposed to the trend of GSI. In November 2018 the mean values of both CIs were found to be the lowest, an increase in December 2018 and a fluctuation in January 2019. This result is explained by the fact that the differences happened within these three months which were triggered by the occurrences of spawning event and the season of gamete release, when compared to a few studies conducted on CI (Jurić et al., 2012; Udoh \& Abiaobo, 2014; Hamli et al., 2017) which particularly affected the values in each month. When eliminating the total weight and using the shell weight, the CI2 values were much smaller compared to CI1 indicating the effect of water bias in the results (Vasconcelos et al., 2008)

On the other hand, the pattern of CI3 using formula of Fdw/Fww x 100 was similar to GSI and it can be concluded to have positive relationship (Hamli et al., 2017). The correlation of positive relationship is shown in Figure 2. When the CI value is low the GSI will have a low value and when the value of $\mathrm{CI}$ is high the value of GSI is also high. A study of CI on other molluscan, the results showed a similar trend of condition index with gonad index and it maybe because of the different stage of gonad which had been collected from the samples (Mladineo et al., 2007) and this factor may explain the relationship of positive correlation between CI and GSI of the black snail for these samples were collected randomly in the water column during the sampling period in Merchang Lagoon. During the research period, in order to get the reading of CI, chemical and physical parameter was taken into account and it seemed that the factors that triggered the abundance (Ikpeze \& Obikwelu, 2016) and the changes in CI value were the seasonal change of weather where the changes alter the weight of $F$. ater flesh, reflecting the health condition. These findings support the idea of changes in CI which correlated with the spawning and resting period being altered by the composition of chemical and physical parameter of the surrounding water as the weather underwent seasonal changes during these three consecutive months (Udoh \& Abiaobo, 2014). In a similar study in Nigeria on gastropod species, researchers analysed the CI and associated the fluctuated value of CI with gamete release period (Udoh \& Abiaobo, 2014).

For GSI, by using formula of $\mathrm{Ggd} / \mathrm{Tww} \mathrm{x}$ 100 the trend differed from CI1, CI2 and CI4 but it has a similar pattern with CI3. From the graph, GSI was at its peak in November 2018 which means its reproductive activity was at the highest condition (Elhasni et al., 2010) while in December 2018 it showed the lowest condition of GSI in the three-month period. There was no significance different between the GSIs (P>0.05). The mean GSI from November 
to December decreasing, but started to increase from December to January 2019. In a previous study, it was said that a decrease in GSI value was related to the gamete release where the gonads were losing weight (Kefi et al., 2014). This explained the results of decreasing gonad weight in December. It determined the period of exertion of gamete (Gharsallah et at., 2010) of $F$. ater being released. Moreover, after the gamete release period, in January 2019, the gonad weight increased but not as high as it was in November 2018. It shows that in November 2018, the gonads were at their best they were was ready for maturity stage where the gamete would be released. Apart from that, in November 2018, an abundance of F. ater was seen in the mangrove area but during December, the snails were observed to be least in number compared to that noted in November when most of the snails were buried in the sand in the water column. During the examination of the gonad and digestive gland section, the obvious differences that could be noted were the weight and the size of the gonad itself. The weight and size of gonad in the period of November and January were much more affirmed compared to what it was in December. The observed changes in GSI of $F$. ater could be attributed to gamete release period according to the recent study (Kefi et al., 2014). However, the main factor might be due to the inability to differentiate between the female and male as there is a lacks references on reproductive status of black snail.

In contrast, the indication of different stages of gonad could not be solidified, mainly because of the method of weighing the gonad and total weight of the black snail which could only detect the differences in the gonadosomatic index not the stages of the gonad, but the possibility of each sample undergoing different phases of gonad development was high as each sample was recorded to had different shell length. According to a finding, the shell length was known to be associated with age, the longest indicated the older the gastropod was (Ismail \& Elkarmi, 2006). To differentiate between the young and the adult individual means, the development of gonad might vary. Thus, some factors may have accumulated to contribute to the changes of the results presented above.

The factors that contributed to the changes could be because they were in synch with the water level or tide (Collin et al., 2017) , as when we were sampling in November, when the tide was low compared to the higher tide in December, and both months were dealing with rainy seasons. The difference was, during sampling in November, the weather was sunny and the temperature was higher, while in December the weather was cloudy, the temperature and the water level were affected by the rain which had stopped few minutes before we reached the sampling site. The results were in synch with those observed in earlier studies on Hexaplex trunculus. It was reported that the spawning activities were altered by the temperature of the water and the duration of daytime (Elhasni et al., 2010).

Despite CI and GSI reading, errors probably occurred where the values of CI changed. Discussion has been made in a paper on condition index of Hexaplex trunculus (Vasconcelos et al., 2008), when processing the samples where proper handling should be taken into account. A fault during the procedure of processing the samples might lead to imprecise data. Apart from that, while cracking the shell of the snails, in order to separate the flesh from it shell, all fragments of the shell need to be distinguished from flesh of the snails. In this stage, some small fragments might be attached to the flesh and some might have cracked and fallen onto the ground and there is where another imprecision of data might have occurred.

The findings of this study have a number of important implications for future practice this study had been carried out in order to enhance a better understanding on condition index and gonadosomatic index of this underexploited species. The results can help future research to compare the similarity of the trends between CI and GSI and to theoretically analyse the relationship between $\mathrm{CI}$ and GSI in these three consecutive months to determine the spawning and gamete release season of black snail, F.ater 
which is important for future research as this species has the potential to be a protein source for the local people in the near future. The following conclusion shows the relationship between CI and GSI. CI can act as an indicator of the spawning session of $F$. ater when compared to the GSI. The results can determine the harvesting period of the species in order to prevent from early extinction of this species when it has a high commercial value (Galvao et al., 2015).

\section{Conclusion}

This study set out to determine the relationship of trend between condition index and gonadosomatic index. The investigation of $F$. ater has shown that there are three methods that seem to be applicable in this experiment which were CI1 CI2 and CI3. They have trend with GSI. The results of this study support the idea that when CI (CI1 and CI2) were at their peaks, the GSI was at it lower condition. The relationship was shown in Figure 2. CI3 has a similar pattern with GSI where the relationship was said to be a positive relationship while for CI1 and CI2 the relationship was said to be negative when compared to the pattern of GSI. In contrast, all three methods have relationship with GSI except for CI4. The condition index and gonadosomatic index methods practically explained the correlation of these two to indicate the spawning and gamete release season. $\mathrm{CI}$ is an alternative way to investigate the reproductive status of the species as the method is more practical and not a time-consuming, thus from the discussion, it has been pointed out that from the relationship of these two where there is positive correlation, for when the CI increased the GSI also increase. Some factors were said to be the main contributions of changing the value of CI in sampling months. It was said that CI can explain the physiological study of an organism but in this case, the numbers of sampling was not enough to consolidate the results. Thus, further research needs to be done to establish the results. Long-term of research might make a great contribution in order to get a better result.

\section{Acknowledgements}

I would like to thank my supervisor, Dr. Roslizawati Binti Ab Lah, for the encouragement, guidance and support in completing my final year project. My biggest gratitude to her for the guidance and the willingness to supervise me for the project, she had given me a lot of help and information despite of her tight schedule. It was an honour to be supervised by her. She taught me many things in carrying out this research. Apart from that, I am thankful to the staff of the Faculty Fisheries and Food Sciences and the laboratory assistance for the guidance and assisting me throughout the project during the sampling period and the laboratory works itself. My thanks to the staff who had willingly sacrificed their time even during weekends to open the laboratory session. To date, all my friends who had been supporting me and giving me suggestions and sharing knowledge on this matter. I would like to thank you a lot. My greatest appreciation goes to my parents who have helped me spiritually and financially to complete this final year project.

\section{References}

Brandt, R. A. M., (1974). The Non-Marine Aquatic Mollusca of Thailand. Archiv für Molluskenkunde, 105, I-IV, 1-423.

Collin, R., Kerr, K., Contolini, G., \& Ochoa, I. (2017). Reproductive Cycles in Tropical Intertidal Gastropods are Timed Around Tidal Amplitude Cycles. Ecology and Evolution, 7(15), 5977-5991.

Das, R. R., Joyson, J., Jeevamani, J., Sankar, R., Samuel, D., \& Kumar, V. (2018). Limited Distribution of Devil Snail Faunus ater (Linnaeus, 1758 ) in Tropical Mangrove Habitats of India. (August).

Elhasni, K., Vasconcelos, P., Ghorbel, M., \& Jarboui, O. (2013). Reproductive Cycle of Bolinus brandaris (Gastropoda: Muricidae) in the Gulf of Gabès (Southern Tunisia). Mediterranean Marine Science, 14(1), 2435. 
Galvao, P., Longo, R., Torres, J. P. M., \& Malm, O. (2015). Estimating the Potential Production of the Brown Mussel Perna perna (Linnaeus, 1758) Reared in Three Tropical Bays by Different Methods of Condition Indices. Journal of Marine Biology, 2015(October).

Gharsallah, I. H., Vasconcelos, P., ZamouriLangar, P., \& Missaoui, H. (2010). Reproductive Cycle and Biochemical Composition of Hexaplex trunculus (Gastropod: Muricidae) from Bizerte Lagoon, Northern Tunisia. Aquatic Biology, 10, 155- 166.

Hamli, H., Idris, M. H., Rajaee, A. H., Kamal, A. H. M., \& Hoque, M. N. (2017). Condition Index of Meretrix lyrata (Sowerby 1851) and Its Relationship with Water Parameter in Sarawak. Sains Malaysiana, 46(4), 545551.

Houbrick, R. S., (1991). Anatomy and Systematic Placement of Faunus Montfort, 1810 (Prosobranchia: Melanopsidae). Malacological Review, 24(1-2), 35-54.

Ikpeze, O. O., \& Obikwelu, M. E. (2016). Factors Affecting Seasonal Abundance of Gastropod of Public Health Importance Found at Agulu Lake Shorelines in Nigeria. International Journal of Pure \& Applied Bioscience),4(2), 91-102.

Ismail, N. S., \& Elkarmi, A. Z. (2006). Age, Growth and Shell Moephometrics of the Gastropod Monodonta dama (Nerithidae:Prosobranchia) from the Gulf of Aqaba, Red Sea. Pakistan Journal of Biological Sciences, 9(5), 843-847.

Jurić, I., Bušelić, I., Balić, D. E.-, Vrgoč, N., \& Peharda, M. (2012). Age, Growth and Condition Index of Venerupis decussata (Linnaeus , 1758 ) in the Eastern Adriatic Sea, 618(November 2007), 613-618.
Kefi, F. J., Boubaker,S., \& El Menif, N. T. (2014). Relative Growth and Reproductive Cycle of the Date Mussel Lithophaga lithophaga (Linnaeus, 1758) Sampled from the Bizerte Bay (Northen Tunisia). Helgoland Marine Research,68(3), 439-450.

Lok, A. F. S. L., Ang, W.F., Ng, P.X., Ng, B. Y.Q., \& Tan, S. K. (2011). Status and Distribution of Faunus ater (Linnaeus, 1758) (Mollusca: Cerithioidea) in Singapore. Nature in Singapore, 4(May), 115-121.

Lucas, A., \& Beninger, P. G. (1985). The Use of Physiological Condition Indices in Marine Bivalve Aquaculture, Aquaculture, 44(3),187-200.

Mladineo, I., Peharda, M., Orhanović, S., Bolotin, J., Pavela-Vrančić, M., \& Treursić, B. (2007). The Reproductive Cycle, Condition Index and Biochemical Composition of the Horse-Bearded Mussel Modiolus barbatus. Helgoland Marine Research, 61(3), 183-192.

Rebelo, M. F., Amaral, M.C., \& Pfeiffer, W.C. (2005). Oyster Condition Index in Crassostrea rhizophorae (Guilding, 1828) from a Heavy-Metal Polluted Coastal Lagoon. Brazilian Journal of Biology, 65(2), 345-351.

Udoh, J. P., \& Abiaobo, N. O. (2014). Condition Index, Meat Yield and Population Structure of the Marine Gastropod, Thais coronata, off Cross River Estuary, Nigeria, 23, 24-32.

Vasconcelos, P., Lopes, B., Castro, M., \& Gaspar, M. B. (2008). Comparison of Indices for the Assessment of Reproductive Activity in Hexaplex trunculus (Gastropoda: Muricidae). Marine Biology Research, 4(5), 392-399. 